\title{
The Effective Potential as an Energy Density: The One Phase Region
}

\author{
Gordon Slade
}

Department of Mathematics, University of Virginia, Charlottesville, VA 22903, USA

\begin{abstract}
An explicit formula is given relating the effective potential in a finite volume $P(\phi)_{2}$ quantum field theory to the expected energy density under the constraint of a fixed average field. In the one phase region, i.e., where the classical potential equals its convex hull and has nonvanishing second derivative, it is shown via a central limit theorem that in the infinite volume limit the effective potential is equal to the constrained energy density, provided $\hbar$ is sufficiently small.
\end{abstract}

\section{Introduction}

The effective potential in a quantum field theory is the Legendre transform of the generator of the connected Feynman vacuum graphs. In [2] it is argued that the physical meaning of the effective potential evaluated at the classical field $a$ is the expected energy density under the constraint that the average field has value $a$. In [6] a heuristic argument using functional integrals is given in support of this physical interpretation. Steps are taken towards making this interpretation rigorous in [9].

Since the constrained energy density is in general not convex in finite volume, while the effective potential is convex, the two quantities cannot in general be equal in finite volume. In Theorem 1 below, an explicit formula is given relating the effective potential of a finite volume $P(\phi)_{2}$ theory to the expected energy density under the constraint of a fixed average field. In Theorem 2 below, it is shown using a central limit theorem that in the infinite volume limit the two quantities are equal in the one phase region, provided $\hbar$ is sufficiently small.

We now introduce the notation. Let $P$ be a polynomial of degree greater than or equal to four which is bounded below, and let $m>0$. For $\mu \in \mathbb{R}$, let

$$
U_{\mu}(a)=P(a)+\frac{1}{2} m^{2} a^{2}-\mu a .
$$

The classical potential of the model is then $U_{0}$. The one-phase region is the complement of the set $B$, defined as follows:

$$
B=\left\{a \in \mathbb{R}: U_{0}(a) \neq\left(\operatorname{conv} U_{0}(a)\right\}^{-} \cup\left\{a \in \mathbb{R}: U_{0}^{\prime \prime}(a)=0\right\},\right.
$$

where conv $U_{0}$ denotes the convex hull of $U_{0}$. Let $d \mu$ be the Gaussian measure on 
$\mathscr{S}^{\prime}\left(\mathbb{R}^{2}\right)$ with mean zero and covariance $\left(-\Delta+m^{2}\right)^{-1}$, where $\Delta$ is the Laplacian on $\mathbb{R}^{2}$ with periodic boundary conditions on the boundary of a rectangle $\Lambda \subset \mathbb{R}^{2}$. The partition function is given by

$$
Z_{\Lambda}(\mu)=\int \exp \left[-\int_{\Lambda}\left(: U_{\mu}(\phi(x)):-\frac{1}{2} m^{2}: \phi(x)^{2}:\right) d x\right] d \mu,
$$

where the Wick dots are with respect to $\left(-\Delta+m^{2}\right)^{-1}$.

The finite volume pressure

$$
p_{\Lambda}(\mu)=\frac{1}{|\Lambda|} \ln \left[Z_{\Lambda}(\mu) / Z_{\Lambda}(0)\right]
$$

is the (negative) expected energy density in the presence of an external field $\mu$. The finite volume effective potential corresponding to the classical potential $U_{0}$ is given by

$$
V_{\Lambda}(a)=\sup _{\mu \in \mathbb{R}}\left[\mu a-p_{\Lambda}(\mu)\right]
$$

where the classical field $a$ is a real parameter. In the infinite volume limit, the pressure is given by

$$
p(\mu)=\lim _{\Lambda \uparrow \mathbb{R}^{2}} p_{\Lambda}(\mu),
$$

(the limit is shown to exist in [8]), and the effective potential by

$$
V(a)=\lim _{\Lambda \uparrow \mathbb{R}^{2}} V_{\Lambda}(a)=\sup _{\mu \in \mathbb{R}}[\mu a-p(\mu)] .
$$

(It is not hard to see that the limit and supremum may be interchanged.)

The dependence of the effective potential on $\hbar$ has not been made explicit, but since we will want to consider small $\hbar$, we point out that $V_{\Lambda}$ depends on $\hbar$ through $p_{\Lambda}$, whose $\hbar$ dependence is obtained by multiplying the covariance in $Z_{\Lambda}$ by $\hbar$, multiplying the interaction in $Z_{A}$ by $\hbar^{-1}$, and multiplying the logarithm in (1.1) by $\hbar$.

Since $p_{\Lambda}$ and $p$ are strictly convex by Hölder's inequality and results of [5] respectively, and since $\pm D_{\mu} p_{\Lambda}(\mu)$ and $\pm D_{\mu} p(\mu)$ can be made arbitrarily large by taking $\Lambda$ and $\pm \mu$ large [12], the suprema in Eqs. (1.2) and (1.3) are attained respectively at the unique $\mu_{\Lambda}(a)$ and $\mu(a)$ satisfying $D_{\mu} p_{\Lambda}\left(\mu_{\Lambda}(a)\right)=a$ and $a \in\left[D_{\mu}^{-} p(\mu(a)), D_{\mu}^{+} p(\mu(a))\right]$, where $D_{\mu}^{( \pm)}$denotes the (right) left derivative with respect to $\mu$. It is not difficult to show that $\lim _{\Lambda \uparrow \mathbb{R}^{2}} \mu_{\Lambda}(a)=\mu(a)$.

Let

$$
d v_{\mu, \Lambda}=Z_{\Lambda}(\mu)^{-1} \exp \left[-\int_{\Lambda}\left(: U_{\mu}(\phi(x)):-\frac{1}{2} m^{2}: \phi(x)^{2}:\right) d x\right] d \mu .
$$

Denote expectations with respect to $d v_{\mu, \Lambda}$ by $\langle\cdot\rangle_{\mu, \Lambda}$ and let

$$
\sigma_{\Lambda}(\mu)=\langle\phi(\Lambda) ; \phi(\Lambda)\rangle_{\mu, \Lambda}^{1 / 2},
$$


where $\phi(\Lambda)=\int_{\Lambda} \phi(x) d x$. Let

$$
D_{\Lambda}(a)=\left\{\phi: \frac{1}{|\Lambda|} \phi(\Lambda)<a\right\} \text { and } A_{\mu, \Lambda}(a)=\left\{\phi: \frac{\phi(\Lambda)-\langle\phi(\Lambda)\rangle_{\mu, \Lambda}}{\sigma_{\Lambda}(\mu)}<a\right\} \text {, }
$$

and define distribution functions

$$
F_{\mu, \Lambda}(a)=\left\langle 1_{D_{\Lambda^{(a)}}}\right\rangle_{\mu, \Lambda} \text { and } G_{\mu, \Lambda}(a)=\left\langle\mathbb{1}_{A_{\mu, \Lambda^{(a)}}}\right\rangle_{\mu, \Lambda} .
$$

These distributions have analytic densities,

$$
f_{\mu, \Lambda}(a)=\frac{d}{d a} F_{\mu, \Lambda}(a) \quad \text { and } \quad g_{\mu, \Lambda}(a)=\frac{d}{d a} G_{\mu, \Lambda}(a)
$$

as can be seen by translating the field in (1.4) to move the $a$ dependence from the characteristic function to the action, see Sect. 4.

Formally $f_{\mu, \Lambda}(a)=\langle\delta((1 /|\Lambda|) \phi(\Lambda)-a)\rangle_{\mu, \Lambda}$, i.e., $-(1 /|\Lambda|) \ln f_{0, \Lambda}(a)$ is the expected energy density under the constraint $(1 /|\Lambda|) \phi(\Lambda)=a$. The main results of this paper are the following two theorems.

Theorem 1. $V_{\Lambda}(a)=-(1 /|\Lambda|) \ln \left[f_{0, \Lambda}(a) / f_{\mu_{\Lambda}(a), \Lambda}(a)\right]$, for all $a \in \mathbb{R}$.

Theorem 2. Fix $a \notin B$. For $\hbar$ sufficiently small,

$$
f_{\mu_{\Lambda}(a), \Lambda}(a) \sim|\Lambda|^{1 / 2} \text { as } \Lambda \uparrow \mathbb{R}^{2} .
$$

In particular, $V(a)=-\lim _{\Lambda \uparrow \mathbb{B}^{2}} \frac{1}{|\Lambda|} \ln f_{0, \Lambda}(a)$.

The proof of Theorem 1 is elementary and is given in Sect. 2. We now discuss the method of proof of Theorem 2. For $a \notin B$ and small $\hbar, U_{\mu_{A}(a)}$ has a uniquely attained global minimum, with positive curvature. Decreasing $\hbar$ corresponds to increasing the difference between the location and value of a local minimum of $\hbar^{-1} U_{\mu_{\Lambda}(a)}\left(\hbar^{1 / 2} \phi\right)$ and the respective quantities for the global minimum (scaling $\phi \rightarrow \hbar^{1 / 2} \phi$ removes the $\hbar$ dependence from the covariance). In this situation, the cluster expansion of [11] (see [4] for related results) applies, and the field variables are weakly dependent in the sense that $\sigma_{\Lambda}\left(\mu_{\Lambda}(a)\right) \sim|\Lambda|^{1 / 2}$. Using this behavior of the standard deviation, a central limit theorem is proved in Sect. 3 for fluctuations of $\phi(\Lambda)$ around its expected value $a|\Lambda|$, i.e., for $G_{\mu_{\Lambda}(a), \Lambda}$. The linear volume divergence of more general truncated correlation functions is used to obtain a local central limit theorem of the form $g_{\mu_{\Lambda}(a), \Lambda}(0) \rightarrow 1 / \sqrt{2 \pi}$. This proves Theorem 2 since

$$
f_{\mu_{\Lambda}(a), \Lambda}(a)=\frac{|\Lambda|}{\sigma_{\Lambda}\left(\mu_{\Lambda}(a)\right)} g_{\mu_{\Lambda}(a), \Lambda}(0) .
$$

Theorem 2 shows that in the one phase region $f_{0, \Lambda}(a) \sim \exp [-V(a)|\Lambda|]$. In the two-phase region different behavior is expected. For example, for small $\hbar$ there is spontaneous magnetization approximately equal to $1 / \sqrt{8}$ in the $P(\phi)_{2}$ model with classical potential $\left(\phi^{2}-1 / 8\right)^{2}[7]$, so $V(a)=0$ on an interval close to $B=[-1 / \sqrt{8}$, $1 / \sqrt{8}]$. It is expected that $-(1 /|\Lambda|) \ln f_{0, \Lambda}(a)$ is a double well whose barrier flattens 
out to $V(a)=0$ in the infinite volume limit. However, the methods of this paper do not apply to the study of the two-phase region.

In the two-dimensional Ising model, numerical computations [10] support asymptotic behavior of the form $\exp \left[-c|\Lambda|^{1 / 2}\right]$ for an analogue of $f_{0, \Lambda}(a)$, in the two-phase region.

\section{Proof of Theorem 1}

The proof uses the approach used in [9]. Fix $a \in \mathbb{R}$ and $\varepsilon>0$, and let $C_{\varepsilon}(a)=$ $\{\phi: a \leqq(1 /|\Lambda|) \phi(\Lambda)<a+\varepsilon\}$. For any $\mu$,

$$
d v_{0, \Lambda}=e^{-\mu \phi(\Lambda)} d v_{\mu, \Lambda} Z_{\Lambda}(\mu) Z_{\Lambda}(0)^{-1},
$$

and hence, if $\mu \geqq 0$ then

$$
\begin{aligned}
e^{-|\Lambda|\left[\mu(a+\varepsilon)-p_{\Lambda}(\mu)\right]}\left\langle\varepsilon^{-1} \mathbb{1}_{C_{\varepsilon}(a)}\right\rangle_{\mu, \Lambda} & \leqq\left\langle\varepsilon^{-1} \mathbb{1}_{C_{\varepsilon}(a)}\right\rangle_{0, \Lambda} \\
& \leqq e^{-|\Lambda|\left[\mu a-p_{\Lambda}(\mu)\right]}\left\langle\varepsilon^{-1} \mathbb{1}_{C_{\varepsilon}(a)}\right\rangle_{\mu, \Lambda} .
\end{aligned}
$$

If $\mu<0$ the upper and lower bounds are reversed. Taking $\mu=\mu_{\Lambda}(a)$ and letting $\varepsilon \downarrow 0$ gives

$$
e^{-|\Lambda| V_{\Lambda}(a)} f_{\mu_{\Lambda}(a), \Lambda}(a) \leqq f_{0, \Lambda}(a) \leqq e^{-|\Lambda| V_{\Lambda}(a)} f_{\mu_{\Lambda}(a), \Lambda}(a) .
$$

\section{The Central Limit Theorem}

Differentiating the equation

$$
F_{\mu, \Lambda}(a)=G_{\mu, \Lambda}\left[\frac{a|\Lambda|-\langle\phi(\Lambda)\rangle_{\mu, \Lambda}}{\sigma_{\Lambda}(\mu)}\right]
$$

with respect to $a$ and setting $\mu=\mu_{\Lambda}(a)$ gives

$$
f_{\mu_{\Lambda}(a), \Lambda}(a)=\frac{|\Lambda|}{\sigma_{\Lambda}\left(\mu_{\Lambda}(a)\right)} g_{\mu_{\Lambda}(a), \Lambda}(0) .
$$

For $\hbar$ small, it follows from results of [11] that $\mu(a)$ (and hence, for large $\Lambda, \mu_{\Lambda}(a)$ ) is close to its classical value. For $a \notin B$ this classical value is the unique $\mu$ such that $U_{\mu}$ attains its uniquely attained global minimum at $a$, and there is positive curvature at that minimum. Hence, for $a \notin B$ and $\hbar$ small, the high-temperature cluster expansion of [11] implies that $\sigma_{\Lambda} \equiv \sigma_{\Lambda}\left(\mu_{\Lambda}(a)\right) \sim|\Lambda|^{1 / 2}$, and as we show below, that $\left|g_{\Lambda}(0)\right|$, $\left|g_{\Lambda}^{\prime}(0)\right|$ and $\left|g_{\Lambda}^{\prime \prime}(x)\right|$ are bounded uniformly in $\Lambda$ and $x$ in a compact set, where here and in the following we use a subscript $\Lambda$ to denote the pair $\mu_{\Lambda}(a), \Lambda$. The fact that $\sigma_{\Lambda} \sim|\Lambda|^{1 / 2}$ will be used to prove a central limit theorem for $G_{\Lambda}(a)$, which together with the bounds on the density $g_{\Lambda}$ will be used to show that $\lim _{\Lambda} g_{\Lambda}(0)=1 / \sqrt{2 \pi}$.

In view of Eq. (3.1), the fact that $\sigma_{\Lambda} \sim|\Lambda|^{1 / 2}$ and $\lim _{\Lambda}(0)=1 / \sqrt{2 \pi}$ proves Theorem 2. In this section we show that $\lim g_{\Lambda}(0)=1 / \sqrt{2 \pi}$ given the above bounds on $g_{\Lambda}$ and in the next section we establish these bounds. 
Proposition 1. Fix $a \notin B$. For $\hbar$ sufficiently small,

$$
\lim _{\Lambda} G_{\mu_{\Lambda}(a), \Lambda}(t)=\frac{1}{\sqrt{2 \pi}} \int_{-\infty}^{t} e^{-x^{2} / 2} d x .
$$

Proof. This type of result is discussed in [1]. As above, we write $G_{\Lambda}$ for $G_{\mu_{\Lambda}(a), A}$, etc. By Taylor's theorem, the characteristic function $\tilde{g}_{A}$ of $G_{A}$ satisfies

$$
\begin{aligned}
\ln \tilde{g}_{\Lambda}(k) & =\ln \int \exp \left[\frac{i k}{\sigma_{\Lambda}}\left(\phi(\Lambda)-\langle\phi(\Lambda)\rangle_{\Lambda}\right)\right] d v_{\Lambda} \\
& =\frac{-k^{2}}{2}-\frac{i}{2} \sigma_{\Lambda}^{-3} \int_{0}^{k}\langle\phi(\Lambda) ; \phi(\Lambda) ; \phi(\Lambda)\rangle_{\mu_{\Lambda}(a)+\left(i k^{\prime} / \sigma \sigma^{\prime}\right),}\left(k-k^{\prime}\right)^{2} d k^{\prime} .
\end{aligned}
$$

Now $\sigma_{\Lambda} \sim|\Lambda|^{1 / 2}$ and

$$
\langle\phi(\Lambda) ; \phi(\Lambda) ; \phi(\Lambda)\rangle_{\mu_{\Lambda}(a)+\left(i k^{\prime} / \sigma_{\Lambda}\right), \Lambda}=O(|\Lambda|),
$$

if $\hbar$ is sufficiently small, by results of [11]. Taking $\Lambda \uparrow \mathbb{R}^{2}$ in Eq. (3.2) gives lim $\tilde{g}_{\Lambda}(k)=e^{-k^{2} / 2}$ (uniformly on compact sets) and hence

$$
\lim _{\Lambda} \int_{-\infty}^{t} g_{\Lambda}(x) d x=\frac{1}{\sqrt{2} \pi} \int_{-\infty}^{t} e^{-x^{2} / 2} d x
$$

Proposition 2. In the setting of Proposition 1, suppose that $\left|g_{\Lambda}(0)\right|,\left|g_{\Lambda}^{\prime}(0)\right|$ and $\left|g_{\Lambda}^{\prime \prime}(x)\right|$ are bounded uniformly in $\Lambda$ and $x$ in a compact set. Then

$$
\lim _{\Lambda} g_{\Lambda}(0)=1 / \sqrt{2 \pi}
$$

Proof. By Fourier inversion,

$$
g_{\Lambda}(0)-1 / \sqrt{2 \pi}=\frac{1}{2 \pi} \lim _{A \rightarrow \infty} \int_{-A}^{A}\left(\tilde{g}_{\Lambda}(k)-e^{-k^{2} / 2}\right) d k .
$$

We show below that $1 / 2 \pi \lim _{A \rightarrow \infty} \int_{-A}^{A} \tilde{g}_{\Lambda}(k)=g_{\Lambda}(0)$ uniformly in $\Lambda$. Thus given $\varepsilon>0, B$ can be chosen large enough that

$$
\left|g_{\Lambda}(0)-\frac{1}{\sqrt{2 \pi}}\right| \leqq \frac{1}{2 \pi} \int_{-B}^{B}\left|\tilde{g}_{\Lambda}(k)-e^{-k^{2} / 2}\right| d k+\varepsilon .
$$

The proposition now follows from the fact that $\tilde{g}_{\Lambda}(k) \rightarrow e^{-k^{2} / 2}$ uniformly on compact sets.

We now verify that $1 / 2 \pi \lim _{A \rightarrow \infty} \int_{-A}^{A} \tilde{g}_{\Lambda}(k) d k=g_{\Lambda}(0)$ uniformly in $\Lambda$. To begin,

$$
\begin{aligned}
\frac{1}{2 \pi} \int_{-A}^{A} \tilde{g}_{\Lambda}(k) d k-g_{\Lambda}(0)= & \frac{1}{\pi} \int_{-\infty}^{\infty}\left(g_{\Lambda}(x)-g_{\Lambda}(0)\right) \frac{\sin A x}{x} d x \\
= & \frac{1}{\pi} \int_{-\alpha}^{\alpha} \frac{g_{\Lambda}(x)-g_{\Lambda}(0)}{x} \sin A x d x \\
& +\frac{1}{\pi} \int_{|x|>\alpha}\left(g_{\Lambda}(x)-g_{\Lambda}(0)\right) \frac{\sin A x}{x} d x \equiv I_{1}+I_{2} .
\end{aligned}
$$


Now,

$$
\begin{aligned}
\left|I_{2}\right| & \leqq \frac{1}{\pi} \int_{|x|>\alpha} \frac{g_{\Lambda}(x)}{|x|} d x+\frac{1}{\pi} g_{\Lambda}(0)\left|\int_{|x|>\alpha} \frac{\sin A x}{x} d x\right| \\
& \leqq \frac{1}{\pi \alpha} \int_{-\infty}^{\infty} g_{\Lambda}(x) d x+\frac{1}{\pi} g_{\Lambda}(0)\left|\int_{|x|>A \alpha} \frac{\sin x}{x} d x\right| .
\end{aligned}
$$

By choosing $\alpha$ large the right-hand side of (3.3) can be made arbitrarily small, independently of $\Lambda$ and $A \geqq 1$, provided $g_{\Lambda}(0)$ is bounded. To deal with $I_{1}$, let

$$
h_{\Lambda}(x)=\frac{g_{\Lambda}(x)-g_{\Lambda}(0)}{x}, \quad x \neq 0 ; g_{\Lambda}^{\prime}(0), x=0 .
$$

Then

$$
\begin{aligned}
\left|I_{1}\right| & =\frac{1}{\pi}\left|\int_{-\alpha}^{\alpha} h_{\Lambda}(x) \sin A x d x\right| \\
& =\frac{1}{\pi}\left|-\frac{1}{A} h_{\Lambda}(x) \cos A x\right|_{-\alpha}^{\alpha}+\frac{1}{A} \int_{-\alpha}^{\alpha} h_{\Lambda}^{\prime}(x) \cos A x d x \mid,
\end{aligned}
$$

which can be made arbitrarily small by choosing $A$ large if $\left|h_{\Lambda}( \pm \alpha)\right|$ and $\left|h_{\Lambda}^{\prime}(x)\right|$ are bounded, $|x| \leqq \alpha$. These bounds follow from the hypotheses on $g_{\Lambda}$.

\section{Bounds on $g_{A}$}

In this section we complete the proof of Theorem 2 by establishing the bounds on $g_{\Lambda}$ used as hypotheses in Proposition 2. As discussed in the last section, there is a convergent cluster expansion for small $\hbar$, and hence [3]

$$
\left\langle: \phi^{k_{1}}(\Lambda): ;: \phi^{k_{2}}(\Lambda): ; \cdots ;: \phi^{k_{r}}(\Lambda):\right\rangle_{\Lambda}=O(|\Lambda|) .
$$

We show how this linear divergence of the truncated correlation functions implies the bounds on $g_{\Lambda}$.

Translation of $\phi$ in $G_{\Lambda}(x)$ by $x \sigma_{\Lambda} /|\Lambda|$, followed by differentiation with respect to $x$ and translation of $\phi$ by $-x \sigma_{\Lambda} /|\Lambda|$, gives

$$
g_{\Lambda}(x)=\frac{\sigma_{\Lambda}}{|\Lambda|}\left\langle\mathbb{1}_{A_{\Lambda}(x)}\left[-\int_{\Lambda}: U_{\mu_{\Lambda}(a)}^{\prime}(\phi):\right]\right\rangle_{\Lambda} .
$$

Further differentiation with respect to $x$ performed before the second translation, followed by translation of $\phi$ gives

$$
\left.g_{\Lambda}^{\prime}(x)=\left[\frac{\sigma_{\Lambda}}{|\Lambda|}\right]^{2}\left\langle\mathbb{1}_{A_{\Lambda}(x)}\left(-\int_{\Lambda}: U_{\mu_{\Lambda}(a)}^{\prime}(\phi):\right)^{2}\right\rangle_{\Lambda}+\left\langle\mathbb{1}_{A_{\Lambda}(x)}\left(-\int_{\Lambda}: U_{\mu_{\Lambda^{(}}(a)}^{\prime \prime}(\phi):\right)\right\rangle_{\Lambda}\right]
$$

and

$$
g_{\Lambda}^{\prime \prime}(x)=\left[\frac{\sigma_{\Lambda}}{|\Lambda|}\right]^{3}\left[\left\langle 0_{A_{\Lambda}(x)}\left(-\int_{\Lambda}: U_{\mu_{\Lambda}(a)}^{\prime}(\phi):\right)^{3}\right\rangle_{\Lambda}\right.
$$




$$
\begin{aligned}
& +3\left\langle\mathbb{1}_{A_{\Lambda}(x)}\left(-\int_{\Lambda}: U_{\mu_{\Lambda}(a)}^{\prime}(\phi):\right)\left(-\int_{\Lambda}: U_{\mu_{\Lambda}(a)}^{\prime \prime}(\phi):\right)\right\rangle_{\Lambda} \\
& \left.+\left\langle\mathbb{1}_{A_{\Lambda}(x)}\left(-\int_{\Lambda}: U_{\mu_{\Lambda}(a)}^{\prime \prime \prime}(\phi):\right)\right\rangle_{\Lambda}\right] .
\end{aligned}
$$

Each term on the right-hand side of (4.2)-(4.4) can be bounded uniformly in $x$ and $\Lambda$ using the Cauchy-Schwarz inequality to separate the characteristic function from the derivatives of $U_{\mu_{\Lambda}(a)}$, Eq. (4.1), and the fact that $\left\langle\int_{\Lambda}: U_{\mu}^{\prime}(\phi):\right\rangle_{\mu, \Lambda}=0$ (because $\left\langle\int_{\Lambda}: U_{\mu}^{\prime}(\phi):\right\rangle_{\mu, \Lambda}$ is proportional to $\left.(d / d x) \int e^{-\int_{\Lambda}\left(: U_{\mu}(\phi+x):-1 / 2 m^{2} \cdot \phi^{2}:\right)} d \mu=(d / d x) Z_{\Lambda}(\mu)=0\right)$. For example,

$$
\begin{aligned}
& {\left[\frac{\sigma_{\Lambda}}{|\Lambda|}\right]^{3}\left|\left\langle 0_{A_{\Lambda}(x)}\left[-\int: U_{\mu_{\Lambda}(a)}^{\prime}(\phi):\right]\left[-\int: U_{\mu_{\Lambda}(a)}^{\prime \prime}(\phi):\right]\right\rangle_{\Lambda}\right|} \\
& \quad \leqq\left[\frac{\sigma_{\Lambda}}{|\Lambda|}\right]^{3}\left\langle 1_{A_{\Lambda}(x)}\right\rangle_{\Lambda}^{1 / 2}\left\langle\left[-\int: U_{\mu_{\Lambda}(a)}^{\prime}(\phi):\right]^{2}\left[-\int: U_{\mu_{\Lambda}(a)}^{\prime \prime}(\phi):\right]^{2}\right\rangle_{\Lambda}^{1 / 2}
\end{aligned}
$$

Using the abbreviation ' $\ldots{ }^{\prime}=-\int_{\Lambda}: U_{\mu_{\Lambda}(a)}^{\prime \prime \prime}(\phi)$ :, the right-hand side of $(4.5)$ is bounded above by

$$
\begin{aligned}
& \left.\left[\frac{\sigma_{\Lambda}}{|\Lambda|}\right]^{3}\left[\left\langle{ }^{\prime \prime} ; ;^{\prime \prime} ;{ }^{\prime \prime}\right\rangle_{\Lambda}+2\left\langle{ }^{\prime \prime}\right\rangle_{\Lambda}\left\langle{ }^{\prime} ; ;^{\prime \prime}\right\rangle_{\Lambda}+2\left\langle{ }^{\prime \prime} ;\right\rangle_{\Lambda}\right\rangle^{\prime \prime} ; ;^{\prime}\right\rangle_{\Lambda} \\
& +\left\langle{ }^{\prime \prime} ;\right\rangle_{\Lambda}\left\langle{ }^{\prime} ;^{\prime}\right\rangle_{\Lambda}+\left\langle{ }^{\prime \prime}\right\rangle_{\Lambda}\left\langle{ }^{\prime \prime}\right\rangle_{\Lambda}\left\langle\left\langle^{\prime} ;{ }^{\prime}\right\rangle_{\Lambda}\right]^{1 / 2} \leqq \text { const, }
\end{aligned}
$$

using (4.1) in the last step.

Acknowledgement. I am grateful to John Imbrie for pointing out an error in an earlier version of this paper.

\section{References}

1. Cassandro, M., Jona-Lasinio, G.: Critical point behavior and probability theory. Adv. Phys. 27, 913-941 (1978)

2. Coleman, S.: Secret symmetry. In: Laws of hadronic matter, proceedings of the eleventh course. International School of Physics Ettore Majorana. Zichichi, A. (ed.) New York: Academic Press 1975

3. Dimock, J.: Asymptotic perturbation expansion in the $P(\phi)_{2}$ quantum field theory. Commun. Math. Phys. 35, 347-356 (1974)

4. Eckmann, J-P.: Remarks on the classical limit of quantum field theories. Lett. Math. Phys. 1, 387-394 (1977)

5. Fröhlich, J., Simon, B.: Pure states for general $P(\phi)_{2}$ theories: construction, regularity and variational equality, Ann. Math. 105, 493-526 (1977)

6. Fukuda, R., Kyriakopoulos, E.: Derivation of the effective potential. Nucl. Phys. B85, 354-364 (1975)

7. Glimm, J., Jaffe, A., Spencer, T.: A convergent expansion about mean field theory I, II. Ann. Phys. 101, 610-669 (1976)

8. Guerra, F., Rosen, L., Simon, B.: Boundary conditions for the $P(\phi)_{2}$ Euclidean quantum field theory. Ann. Inst. H. Poincaré 25, 231-334 (1976)

9. Jona-Lasinio, G.: Large fluctuations of random fields and renormalization group: Some perspectives. In: Scaling and self-similarity in physics. Fröhlich, J. (ed.) Boston, Basel, Stuttgart: Birkhäuser 1983 
10. Schulman, L.: Magnetization probabilities and metastability in the Ising model. J. Phys. A: Math. Gen. 13, 237-250 (1980)

11. Slade, G.: The loop expansion for the effective potential in the $P(\phi)_{2}$ quantum field theory. Commun. Math. Phys. 102, 425-462 (1985)

12. Spencer, T.: The mass gap for the $P(\phi)_{2}$ quantum field model with a strong external field. Commun. Math. Phys. 44, 143-164 (1975)

Communicated by K. Osterwalder

Received August 9, 1985; in revised form November 22, 1985 